\title{
Antibody Repertoire
}

National Cancer Institute

\section{Source}

National Cancer Institute. Antibody Repertoire. NCI Thesaurus. Code C19587.

A data set that both identifies the isotype of antibodies and quantifies their concentration

in a biological sample or specimen. 